\title{
High accuracy transportable selectable-Value High Dc Voltage Standard
}

\author{
F. Galliana ${ }^{1}$, R. Cerri ${ }^{2}$ and L. Roncaglione Tet $^{3}$ \\ 1, 2,3 National Institute of Metrological Research, strada delle Cacce 91, 10135 TURIN - Italy \\ 1 +39 0113919336, f.galliana@inrim.it \\ 2+390113919426, r.cerri@inrim.it \\ 3+39 0113919316, I.roncaglione@inrim.it
}

\begin{abstract}
Resumé. À l'Institut national de recherche metrologique (INRIM) il a été développé un étalon de haute tension continue avec des valeurs sélectionnables de $10 \mathrm{~V}$ à $100 \mathrm{~V}$ pour compenser le manque d'étalons de haut niveau de tension continue d'une valeur supérieure à $10 \mathrm{~V}$ pour les comparaisons interlaboratoires de haut niveau. Il a été utilisé une nouvelle technique électronique de terre mobile. L'étalon développé a un bruit inférieur et il a la stabilité égal à celle des calibrateurs de tension continue ou multifonction de haut niveau largement utilisé dans les laboratoires d'étalonnage électriques; il a aussi une meilleure attitude à être transporté pour les comparaisons interlaboratoires. Le projet est extensible jusqu'à $1000 \mathrm{~V}$.
\end{abstract}

\section{Introduction}

The national DC Voltage Standard is today reproduced from the National Standard of Time, through the Josephson effect [1]. The maintenance of the National Standard is granted by groups of Zener-diode-based Dc Voltage Standards whose values are periodically updated repeating the Josephson effect [1-3]. These standards are also excellent transport standards due to their resistance to physical shocks, temperature changes and battery operation mode. For this reason they are also used for the "artifact calibration" with which Digital Multimeters (DMMs) and Multifunction Calibrators (MFCs), can be calibrated and adjusted [4-6]. In addition, for their easy transportability, they are also involved in Interlaboratory comparisons (ILCs). Zener-Dc Voltage Standards were involved both in International and National high level (ILC's. [7-10]. A lack in availability of high performance DC Voltage Standards also for ILC's exists at voltages upper than $10 \mathrm{~V}$. DC Voltage calibrators and MFC's are now the most employed Reference Standards for DC Voltages up to $1000 \mathrm{~V}$. They assure high stability and accuracy, remote control and commercial availability. On the other hand, they can sometimes suffer of noise problems at their input stage [11] and can be damaged during transports due to their dimensions and sensitivity to mechanical stresses. For this reason the risk to use these instruments in multilateral ILCs, where the traveling standards have to perform several trips and in different transport conditions, can be considerably high. To overcome these problems, at National Institute of Metrological Research (INRIM) a modular Multi Value High Accuracy Transportable High DC Voltage Standard (THVS), operating from $10 \mathrm{~V}$ to $100 \mathrm{~V}$ was realized, with the possibility to operate both connected to mains (with an internal net filter or in floating battery mode avoiding noises and with a novel ground mobile electronic developmnent technique. This paper shows the main features of the THVS, its its characterization results also comparing the accuracy and stability of its voltages values with the main commercial top class DC Voltage calibrators and MFCs in its most critical operating value $(100 \mathrm{~V})$, its calibration and use uncertainties as local or transportable Standard.

\section{Description of the THVS}

Although DC Voltage calibrators and MFCs are top class instruments, in some applications noise problems can arise. For example, when in a measurement circuit other sensitive instruments are involved besides them, common mode and power supply noises may lead to measurement errors. In particular at higher voltages noises and disturbs of DC Voltage calibrators and MFCs can be significant due to their many internal circuits [11]. An attempt to reduce these problems was tried with the employed technique for the development of the THVS that was projected to operate disconnected from mains as all its circuits can be supplied by means of a set of lead batteries that are recharged when the device is not under measure.

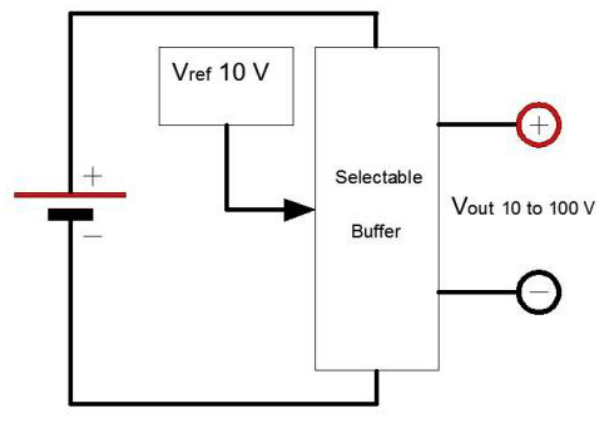

Figure 1. Block scheme of the THVS.

f.galliana@inrim.it 
The THVS is a DC Voltage source that can provide DC settable Voltages ranging from $10 \mathrm{~V}$ to $100 \mathrm{~V}$. It has an a circuit having the function of internal reference voltage (Output Voltage: 10 Vdc, Temperature Coefficient: (TCR) $\quad<0.003 \times 10^{-6} /{ }^{\circ} \mathrm{C}$, stability $\pm 0.5 \times 10^{-6} /$ year). A principle scheme of the THVS is shown in Fig. 2 .

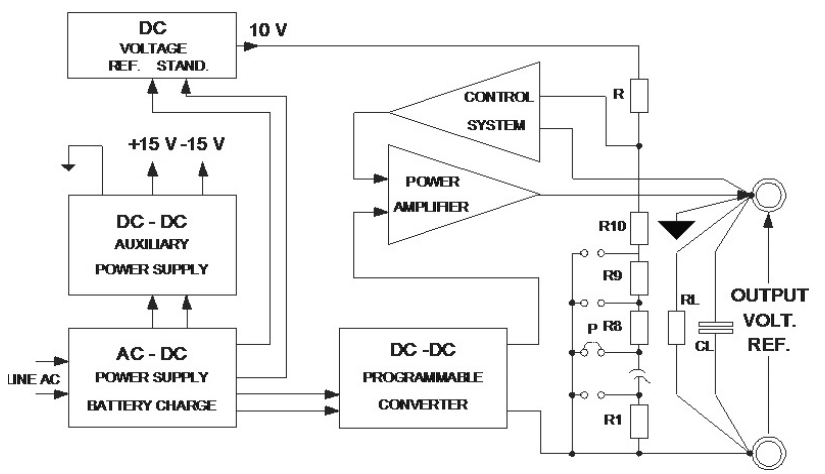

Figure 2. THVS principle scheme.

The THVS, to provide the desired voltages, receives the correct supply voltages by means of precision lowripple programmable DC-DC converters with proper output voltage to obtain the desired output voltage. An additional low noise and high insulation capacity DC-DC converter switching with outputs of $\pm 15 \mathrm{Vdc}$ generate auxiliary voltages to control the output stage,. This stage employs high voltage P-channel MOS components as power buffer. The control circuits were made with precision operational amplifiers with very low offset and low TCR. The reference resistors wee hermetically sealed ultra-high-precision Z-Foil with TCR lower than $0.4 \times 10^{-}$ ${ }^{6} /{ }^{\circ} \mathrm{C}$. The output stage is equipped with a protection system for maximum voltage and current. A novel assembly method, based on a ground-mobile technique, was adopted allowing to control the generated voltages with active components normally used for low voltages available at lower cost. It is shown in Fig. 2 where it is visible that the ground potential is driven to the high potential. Excluding some auxiliary circuits for control of the battery pack, all the electrical power required by the circuitry to control the electrical output of which the THVS is composed, is provided integrally by its rechargeable lead batteries. In fact, three lead $6 \mathrm{~V} 5 \mathrm{Ah}$ rechargeable batteries ensure to the instrument ann autonomous operation for 36 hours. Downstream the batteries, an accurate voltage regulator provides a DC voltage of $12 \mathrm{~V}$ needed to the remaining electronic components. Its characteristics are:Vout: $12 \mathrm{~V}$, noise voltage of $30 \mu \mathrm{Vpp}$, TCR of $1 \times 10^{-5} /{ }^{\circ} \mathrm{C}$ and output current of $0.5 \mathrm{~A}$. The project is also suitable to be upgraded in future adding to the actual realization, a module with a Voltage source providing a a DC settable Voltage ranging from $200 \mathrm{~V}$ to $1000 \mathrm{~V}$.

\subsection{THVS components and characteristics}

The resistors involved in the THVS are Vishay VSRJ type $10 \mathrm{k} \Omega$ with tolerance of $\pm 0.05 \%$ ), TCR lower than $0.4 \times 10^{-6} /{ }^{\circ} \mathrm{C}$, thermal electromotive force (EMF) of $\pm 0.05 \mu \mathrm{V} / \mathrm{V}$, power at $70^{\circ} \mathrm{C}$ of $\cong 0.3 \mathrm{~W}$ except in the case of $10 \mathrm{~V}$ and $20 \mathrm{~V}$ in which two Vishay VH102Z type $10 \mathrm{k} \Omega$ with same tolerance and power but with TCR lower than $0.2 \times 10^{-6} /{ }^{\circ} \mathrm{C}$ were inserted to improve the stability of the two lowest values of the THVS.

The main electronic component is a MOSFET VISHAY mod. IRFR220 with $\mathrm{V}_{\text {drain-Source(DS): }} 200 \mathrm{~V}, \mathrm{R}_{\mathrm{DS}}$ on of $0.8 \Omega$, IDSon: $3 \mathrm{~A}$. This power stage realized with technologies Hexfet (Power MOSFET) designed to operate at constant power regardless of the set output voltage;

- a DC / DC switching with programmable output from 18 to $110 \mathrm{Vdc}$ with stability: $<25 \times 10^{-6} /{ }^{\circ} \mathrm{C}$, peak to peak ripple at full load: $<0.01 \%$, frequency $80 \mathrm{kHz} \div 180 \mathrm{kHz}$ and high insulation capacity;

- a control circuit for the power section made with ultraprecision operational amplifier (Offset voltage: $<10 \mu \mathrm{V}$, offset drift: $<0.1 \mu \mathrm{V} /{ }^{\circ} \mathrm{C} \max$, Open-loop gain of $12 \mathrm{~V} / \mu \mathrm{s}$.

\subsubsection{Specifications of the THVS.}

The specifications of the THVS are:

- Output voltages: from $10 \mathrm{~V}$ to $100 \mathrm{~V}$ selectable by means of an external switching system placed on the front panel by means of deep switches; output currents $\geq 5 \mathrm{~mA}$;

- Output noise at $100 \mathrm{~V}$ of about $106 \mu \mathrm{V}$ rms vs. $155 \mu \mathrm{V}$ and $153 \mu \mathrm{V}$ of a top class DC Voltage Calibrator and a top class MFC as declared by the manufacturers;

- Evaluated $24 \mathrm{~h}$ mean stability of $5.6 \times 10^{-8}$ at $100 \mathrm{~V}$ to compare with the $24 \mathrm{~h}$ evaluated mean stability of $9.5 \times 10^{-8}$ and $1.4 \times 10^{-7}$ of two top class DC Voltage Calibrators and two MFCs at $100 \mathrm{~V}$;

- possibility to operate connected to mains or in floating mode disconnected from mains.

\subsubsection{Thermal features of the THVS.}

The THVS has a thermal compensator that maintains the temperature inside it at $37.70{ }^{\circ} \mathrm{C}$ rejecting the temperature changes due to different load effects due to the different voltages and due to external temperature variations. The action of this compensator allows a better stability and sensitively reduces the waiting times to start the measurements after a voltage change. By means of this thermal compensator, the maximum temperature fluctuations in the THVS in an electrical Laboratory is $\pm 0.15{ }^{\circ} \mathrm{C}$ lowering the relevant uncertainty component. In addition, as the THVS is maintained always this temperature, its humidity dependence is minimized.

\section{Comparison with DC Voltage calibrators and MFC's}

Two alternative tests were carried out to compare the THVS at $100 \mathrm{~V}$ with high accuracy DC Voltage Calibrators and MFCs in their DC Voltage mode. In the first test, the THVS, a DCV Calibrator and a MFC were compared connecting them alternatively to the same high 
accuracy DMM in its $100 \mathrm{~V}$ range. The DMM measurements at $100 \mathrm{~V}$ were computed nulling its $0 \mathrm{~V}$ readings. The measurements were made in a shielded laboratory thermoregulated at $(23 \pm 0.3){ }^{\circ} \mathrm{C}$ and at a relative humidity of $(40 \pm 10) \%$. The $12 \mathrm{~h}$ measurement obtained results of the three standards are shown in Fig. 3. In this case the THVS was fed by a generator connected to the mains. The measurements spreads, evaluated as standard deviations of the measurements, were $5.8 \times 10^{-8}, 1.0 \times 10^{-7}$ and $8.3 \times 10^{-8}$ respectively for the THVS, for the DCV Calibrator and for the MFC. These values included the DMM contribution that was considered stable in the three evaluations as the better available one was selected. The lowest drift was obtained by the DCV Calibrator. In Fig. 4 the $3 \mathrm{~h}$ measurement behavior of all the DC Voltages provided by the THVS are reported. The lowest spreads were obtained at $10 \mathrm{~V}$ and in particular at $20 \mathrm{~V}$ (respectively $4.9 \times 10^{-8}$ and $1.0 \times 10^{-8}$, probably due to the better features of their 10 $\mathrm{k} \Omega$ resistors .

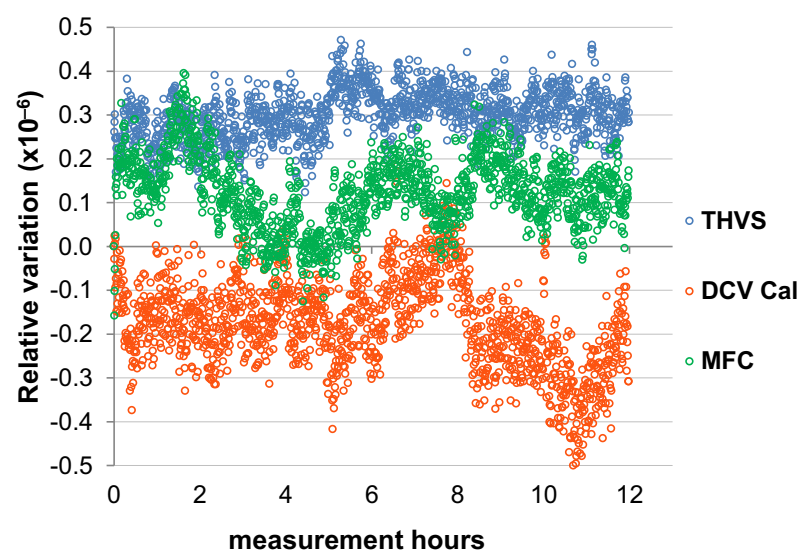

Figure 3. $12 \mathrm{~h}$ spread and drift comparison among the THVS, a DCV Calibrator and a MFC at $100 \mathrm{~V}$ reading with a high accuracy DMM.

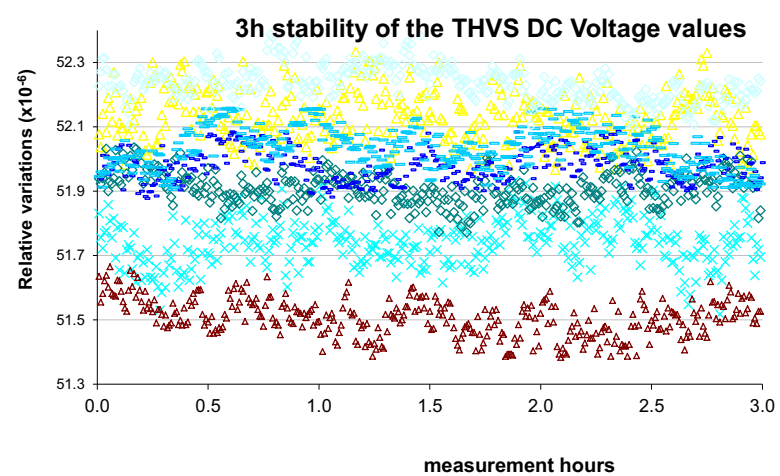

Figure 4. 3h stability comparison among the DC Voltages that the THVS can provide.

With the second test three DMMs with similar noise and intrinsic repeatability were selected. This selection was made connecting them at the same time, in their DC Voltage mode, to a high stability same DCV source, and evaluating their measurements repeatability. Then, the THVS, two top class DCV calibrators and MFCs were compared at $100 \mathrm{~V}$ in a $24 \mathrm{~h}$ time-period after a same stabilization period. The measurements were carried out during the weekend to avoid disturbs due to presence of the operators. In this case the three instruments under comparison underwent the same environmental fluctuations. Figs 5, 6 show the obtained results. the THVS was battery-fed and with the voltage battery regulator. In the $24 \mathrm{~h}$ measurements with the three DMM test the spreads were $5.7 \times 10^{-8}, 1.2 \times 10^{-7}$ and $6.8 \times 10^{-8}$ respectively for the THVS and for the two DCV Calibrators. The lowest drift was reached by the $\mathrm{DCVCal}_{1}$ with a relative drift of $1.4 \times 10^{-9} / \mathrm{h}$ vs $2.6 \times 10^{-9} / \mathrm{h}$ and $1.4 \times 10^{-8}$ for the THVS and the $\mathrm{DCVCal}_{2}$.

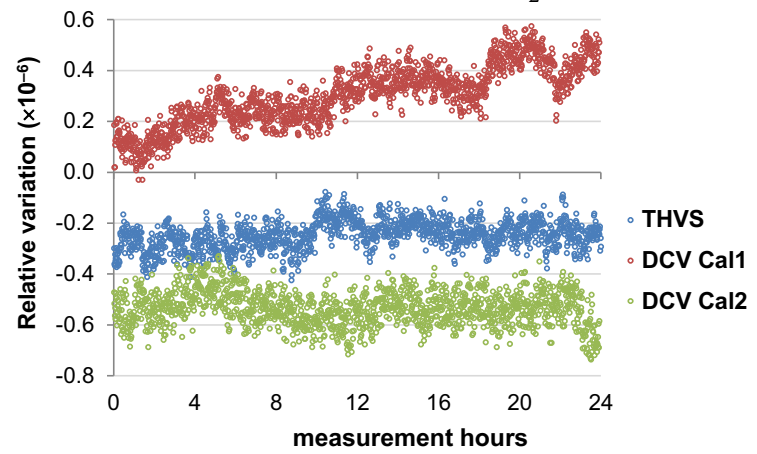

Figure 5. 24h spread and drift of the THVS and of two DCV Calibrators at $100 \mathrm{~V}$ with the three DMMs test.

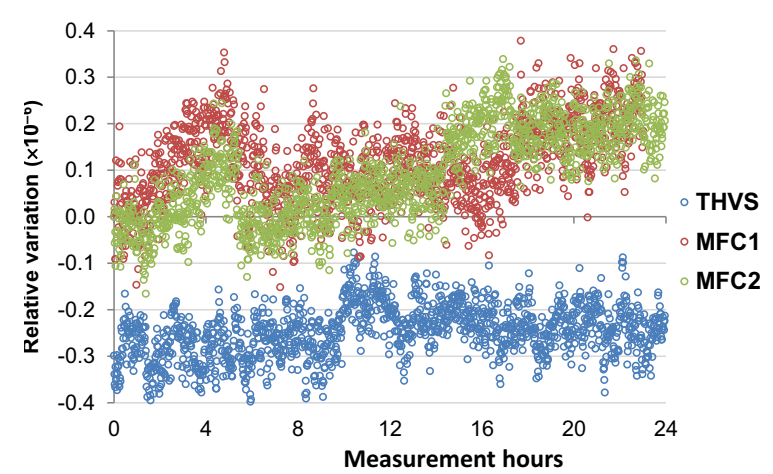

Figure 6. $24 \mathrm{~h}$ spread and drift of the THVS and of two MFCs at $100 \mathrm{~V}$ with the three DMMs test.

The same test was repeated with the THVS and two MFCs. In this test the spreads were $5.6 \times 10^{-8}, 2.3 \times 10^{-7}$ and $1.0 \times 10^{-7}$ respectively for the THVS and for the two MFCs. The lowest drift was reached by the THVS with a relative drift of $2.7 \times 10^{-9} / \mathrm{h}$ vs. $5.0 \times 10^{-9} / \mathrm{h}$ and $1.1 \times 10^{-8} / \mathrm{h}$ for the two MFCs.

\section{Calibration of the THVS}

The measurement setup shown in Fig. 7 is used for the THVS calibration. The THVS is calibrated with an opposition method connecting it to the input of a high accuracy INRIM calibrated Voltage Divider [12] set in suitable ratio and compared it with the $10 \mathrm{~V}$ standard value of a INRIM Zener DC Voltage Standard calibrated vs. the INRIM National Standard Voltage connected through a high accuracy DMM used as nano-voltmeter to the output of the Divider. 


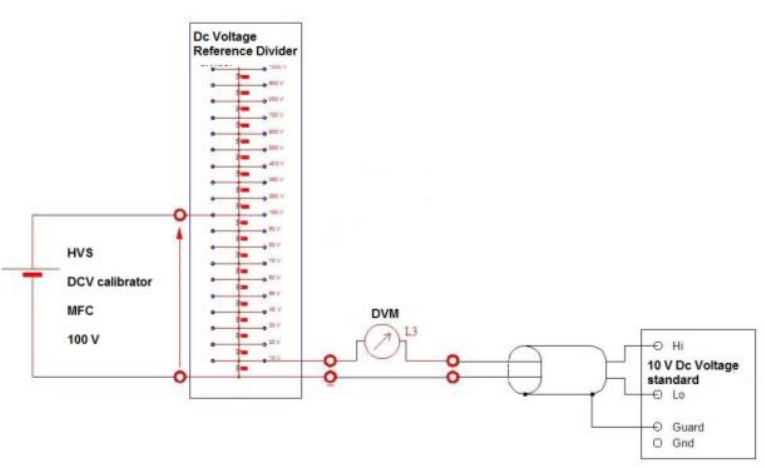

Figure 7. Measurement setup to calibrate the THVS.

Hence the THVS value is then:

$$
V_{\text {THVS }}=\frac{V_{s}+v}{D}
$$

Where $V$ is the $10 \mathrm{~V}$ DC Voltage of the $10 \mathrm{~V}$ Standard, $v$ the voltage unbalance and $D$ the Divider Voltage ratio. In Fig. 8 a photo of the measurement setup of fig. 7 is shown.

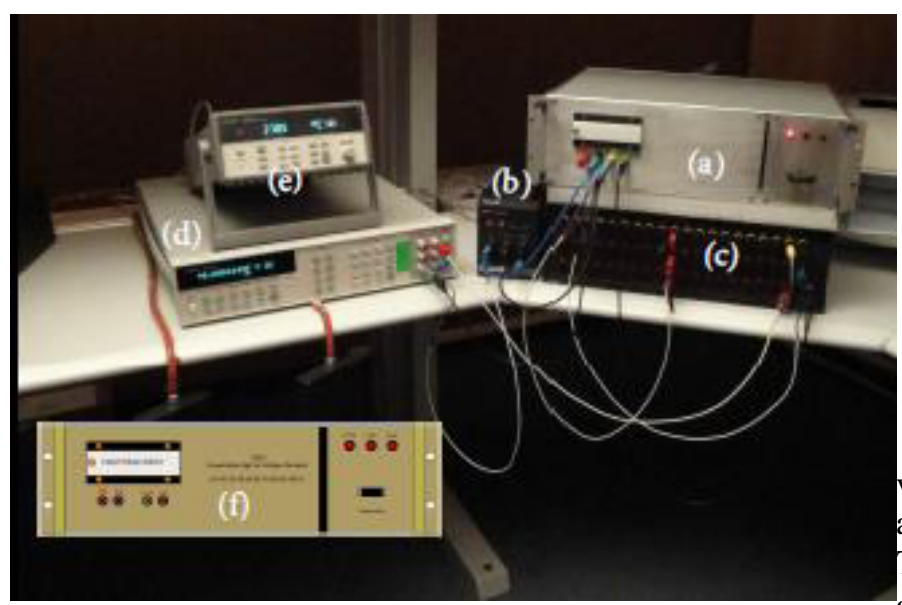

Figure 8. View of the measurement setup to calibrate the THVS. a) THVS, b) $10 \mathrm{~V}$ Reference Standard. c) Dc voltage divider, d) DMM used to evaluate the unbalance voltages, e) temperature-meter to acquire the temperature inside the THVS and f) screen printing to be successively applied on the THVS front panel.

\section{Evaluation of the uncertainties of the THVS.}

\subsection{THVS calibration uncertainty}

According to the paragraph 4 and to (1) in Table, 1 an uncertainty budget for the calibration of the THVS at 100 $\mathrm{V}$ is given.

Table 1. THVS calibration uncertainty budget at $100 \mathrm{~V}$.

\begin{tabular}{|c|c|c|}
\hline Source & type & $\mathbf{1 \sigma}\left(\times 10^{-7}\right)$ \\
\hline Ref 10 V calibration & $\mathrm{B}$ & 2.5 \\
Ref drift & $\mathrm{B}$ & 1.2 \\
\cline { 2 - 3 } Ref temp. dependence & $\mathrm{B}$ & 0.2 \\
\hline
\end{tabular}

\begin{tabular}{|c|c|c|}
\hline Ref humidity dependence & B & negl. \\
DMM accuracy & B & 0.2. \\
DMM calib. & B & 0.1 \\
Unbal. repeatability & B & 0.9 \\
Divider calib. & A & 1.0 \\
Divider drift & B & 0.1 \\
Divider temp. dependence & B & 0.1 \\
\hline Total RSS
\end{tabular}

For a $95 \%$ confidence level the calibration uncertainty of the THVS at $100 \mathrm{~V}$ is then about $6.2 \times 10^{-7}$. According to these uncertainty components, in Table 2 the expanded calibration uncertainties of the THVS are summarized.

Table 2. THVS calibration uncertainty for each voltage.

\begin{tabular}{|c|c|}
\hline $\begin{array}{c}\text { Voltage } \\
(\mathrm{V})\end{array}$ & $\begin{array}{c}\text { Expanded uncertainty } \\
\left(\times 10^{-7}\right)\end{array}$ \\
\hline 10 & 7.5 \\
20 & 6.6 \\
30 & 6.8 \\
40 & 6.6 \\
50 & 6.6 \\
60 & 6.3 \\
70 & 6.5 \\
80 & 6.7 \\
90 & 6.4 \\
100 & 6.2 \\
\hline
\end{tabular}

\subsubsection{Mid-term stability of the THVS}

Since its assembly, the THVS was also measured at various voltages with the measurement setup of Fig. 7 about every week to evaluate its mid-term stability. The THVS showed a smooth linear decreasing drift since its assembly of a mean value of $6.0 \times 10^{-8} /$ day). The drift behaviour seems justified as a complete stabilization of the THVS internal components has not yet reached. The measurements will continue to detect its regimen drift and its long-term drift.

\subsubsection{Transport effect.}

The transport effect was evaluated transporting the THVS simulating the case in which the it could be transported from INRIM to an external Laboratory. The THVS could be transported by car, van, or plane and maintained for several hours or some days in not controlled temperature conditions till to the arrival to the laboratory. For our test, the THVS was transported in a suitable package by car with $2-3 \mathrm{~h}$ of travel, successively maintained in uncontrolled temperature condition for at least $24 \mathrm{~h}$. Then, the measurements were made in a thermo-regulated laboratory $24 \mathrm{~h}$ after. The observed maximum relative measurement deviation analysing all the voltages was $1.5 \times 10^{-6}$.

\subsection{THVS use uncertainty}

Use uncertainty can be defined as the best uncertainty that the THVS can assure in the time period between two calibrations. in Table 3 a preliminary use uncertainty budget of the THVS use uncertainty at $100 \mathrm{~V}$ is given. It 
was assumed to use the THVS as DC Voltage Standard for 90 days without recalibration.

Table 3. THVS mid-term use uncertainty budget at $100 \mathrm{~V}$.

\begin{tabular}{|c|c|c|}
\hline Source & type & $\mathbf{1 \sigma}\left(\times 10^{-7}\right)$ \\
\hline calibration & $\mathrm{B}$ & 3.0 \\
drift & $\mathrm{B}$ & 12 \\
\hline Temp/hum & & \\
dependence & $\mathrm{B}$ & 0.0 \\
noise & $\mathrm{B}$ & 0.3 \\
\hline Total RSS & \multicolumn{1}{|c|}{$\mathbf{1 2 . 3}$}
\end{tabular}

For a $95 \%$ confidence level the use uncertainty of the THVS at $100 \mathrm{~V}$ is then about $2.5 \times 10^{-6}$. According to the uncertainty components, in Table 4 the expanded use uncertainties of the THVS are summarized. These uncertainty values are valid considering to use the THVS as Laboratory Standard for at least three months after calibration.

Table 4. THVS use uncertainties as laboratory Standard for each voltage

\begin{tabular}{|c|c|}
\hline $\begin{array}{c}\text { Voltage } \\
(\mathrm{V})\end{array}$ & $\begin{array}{c}\text { Expanded uncertainty } \\
\left(\times 10^{-6}\right)\end{array}$ \\
\hline 10 & 4.4 \\
20 & 3.7 \\
30 & 3.5 \\
40 & 4.0 \\
50 & 4.8 \\
60 & 3.0 \\
70 & 2.0 \\
80 & 2.6 \\
90 & 2.7 \\
100 & 2.5 \\
\hline
\end{tabular}

In Table 5 are reported the use uncertainties as traveling Standard, simply adding the transport uncertainty component.

Table 5. THVS use uncertainties as travelling Standard for each voltage.

\begin{tabular}{|c|c|}
\hline $\begin{array}{c}\text { Voltage } \\
(\mathrm{V})\end{array}$ & $\begin{array}{c}\text { Expanded uncertainty } \\
\left(\times 10^{-6}\right)\end{array}$ \\
\hline 10 & 4.5 \\
20 & 3.8 \\
30 & 3.6 \\
40 & 4.1 \\
50 & 4.9 \\
60 & 3.1 \\
70 & 2.1 \\
80 & 2.8 \\
90 & 2.8 \\
100 & 2.6 \\
\hline
\end{tabular}

\section{Conclusions}

In the characterization and stability tests the developed THVS showed lower noise, short and midtime stability and measurement repeatability on the order of top class DC Voltage calibrators and MFCs. This is a significant result as the THVS is not actively thermoregulated. The results show that the THVS is suitable to act as DC Voltage Laboratory top level Voltage Standard or travelling multiple Standard for national ILCs. Future aim will be the evaluation of the THVS pressure dependence to also involve the THVS as Transportable Voltage Standard also for high level International ILCs.

\section{References}

1. Pöpel R., Metrologia, 29 pp. 153-174, (1992).

2. Huntley L.: A, IEEE Trans. Instr. Meas., IM-36,. 4, pp. 908-912, (1987).

3. Witt T.J, Proc. of IEEE Science, Measurement and Technology, 149, 6, pp. 305-312, (2002).

4. Fluke Corporation, Calibration: Philosophy in Practice, Second Edition.

5. G. Rietveld, Artifact calibration: An evaluation of the Fluke 5700A series II calibrator, Rep. ISBN 909\0013\0322-4, (1999).

6. Capra P.P, Galliana F., arXiv:1505.04398v1 [physics.ins-det, (2015).

7. D. Reymann et al., Proc. of the Prec. El. Measur. Conf, pp. 253-254, (2000).

8. Hamilton C. A" IEEE Trans. Instr. Meas., 54, 1, pp. 215-221, (2005).

9. Graetsch V., Staben E., Bachmair H. , Proc. of the Prec. El. Measur. Conf, pp. 14-15, (1988).

10. Latika S.R Becker, Bruce F. Field, Thomas E. Kiess, IEEE Trans. on Instrum. and Meas., Vol. IM-35 4, pp. 383-386, (1986).

11. Callegaro L, D’Elia V., Capra P. P.,. Sosso A, , IEEE Trans. Instr. Meas., 56, 2, pp. 295-299, (2007).

12. Marullo Reedtz G., Cerri R., IEEE Trans. on Instrum. and Meas., 48, 2, pp. 338-341, (1999). 